\title{
Pyridoxamine: another vitamin for sickle cell disease?
}

\author{
Marilyn J. Telen \\ Wellcome Professor of Medicine, Duke University, Durham, NC, USA \\ E-mail: MARILYN J.TELEN - marilyn.telen@duke.edu
}

doi:10.3324/haematol.2020.257998

T he last few years have seen a flurry of activity in the development of pharmacotherapeutics for sickle cell disease (SCD). These efforts have involved the invention and discovery of novel compounds, the development of biologics, and the repurposing of previously available drugs and compounds (reviewed by Telen et al. ${ }^{1}$ ). Against this backdrop, Li et al. now present data regarding the possible utility of pyridoxamine, a form of vitamin $\mathrm{B} 6$, in preventing vasoocclusion and inflammation in SCD. ${ }^{2}$ Vitamin B6 is a term referring to any one or more of 6 vitamers alone or in combination, including pyridoxal and its phosphorylated ester pyridoxal 5' phosphate (and its monohydrate salt), pyridoxine and pyridoxamine, and both of their phosphorylated esters. These six forms are readily interconverted in the body, and pyridoxal 5' phosphate is an essential cofactor in numerous enzymatic reactions in various tissues. When any phosphorylated form of vitamin B6 is ingested, it is usually hydrolyzed by intestinal phosphatases, and the non-phosphorylated forms are then rapidly absorbed. After absorption, the vitamin can then be phosphorylated and converted into the active form. Historically, pyridoxamine was marketed as a dietary supplement, often as the hydrochoride salt, pyridoxamine dihydrochloride. Pyridoxamine became of interest to diabetologists and those studying complications of diabetes because it inhibits the formation of advanced glycation end products (AGE) from glycated proteins and traps pathogenic reactive carbonyl compounds (Amadori reaction products), which are intermediates in the formation of AGE. In 2009, the US Food and Drug Administration designated pyridoxamine as a pharmaceutical, when it became the active ingredient in Pyridorin, a drug designed by Biostratum Inc., and investigated for possible utility in diabetic nephropathy due to its ability to decrease the production of advanced glycation end products (AGE) in animal models of diabetes. ${ }^{3}$ However, a clinical study produced disappointing results, ${ }^{4}$ and studies of vitamin B6 for diabetic nephropathy registered at clinicaltrials.gov appear no longer active. A Cochrane review of available data in 2015 failed to find evidence for improvement of kidney function with vitamin B6 or its derivatives, ${ }^{5}$ despite several animal studies suggesting benefit.

Nonetheless, one report associated plasma levels of advanced glycation end products (pentosidine, $\mathrm{N}$ (epsilon) -(carboxymethyl)lysine (CML) and N(epsilon) -(carboxyethyl)lysine (CEL) with SCD organ complications thought to be hemolysis-related. ${ }^{6}$ At steady state, both pentosidine and CML levels correlated significantly to hemolytic rate, and pentosidine was significantly related to the number of organ complications. Those investigators suggested that increased plasma AGE levels in sickle cell patients might be implicated in the pathophysiology of the hemolytic phenotype of SCD, with its attendant organ damage.

\section{Vitamins for sickle cell disease}

Clinical trials currently listed on the clinicaltrials.gov website include 27 studies involving vitamins and nutritional supplements for patients with SCD. Of these, nine list a form of vitamin $\mathrm{D}$ as the active (but not always the only active) subject of study. Other trials focus on vitamin A, folic acid (with and without other supplements), vitamin E, niacin, nicotinamide, omega-3 fatty acids, sodium bicarbonate, zinc, $\alpha$-lipoic acid, L-carnitine, and ready to use supplementary food (RUSF), which may contain various protein sources, multiple vitamins, calcium, arginine and citrulline. In many cases, the goal of these therapies is to improve response to oxidative stress, which is markedly increased in SCD.

\section{Multiple targets}

When viewing the drug development landscape for SCD, most notable, perhaps, is the wide range of therapeutic targets of the newly approved drugs and drugs still in development. Of the three recently approved drugs for SCD, each has a different therapeutic target. One, L-glutamine, is a nutritional supplement thought to act by reducing the sequelae of oxidative stress. The second, voxelotor, is an anti-polymerization and anti-sickling agent $t^{8}$ that increases the oxygen affinity of hemoglobin and, by hampering deoxygenation, forestalls sickling and hemolysis and usually raises hematocrit. The third, crizanlizumab, ${ }^{9}$ inhibits P-selectin interactions and is thought thereby to reduce adhesive and inflammatory cell-cell interactions. Of these three, two, L-glutamine and crizanlizumab, were approved as a consequence of their efficacy in reducing the frequency of vaso-occlusive events, while the third, voxelotor, was approved due to its ability to improve anemia by reducing hemolysis, without regard to any effect on vaso-occlusive events. Disappointingly, none of the studies leading to approval of these drugs involved extensive exploration of the pathophysiologic mechanism affected. In contradistinction, the current paper by Li et al. has explored many mechanistic aspects of potential pyridoxamine effect. ${ }^{2}$

\section{Targeting oxidative stress}

An increasing body of literature supports the concept that oxidative stress is an important contributor to the pathophysiology of SCD, including the phenomena of hemolysis, vaso-occlusion and multi-organ damage..$^{10-13}$ There are multiple mechanisms that tie vaso-occlusion in SCD to oxidative stress and oxidative damage (Figure 1). Pyridoxamine has been shown to facilitate reduction in oxidative stress parameters and reactive oxygen species (ROS) production. ${ }^{14}$ At physiological $\mathrm{pH}$, pyridoxamine 


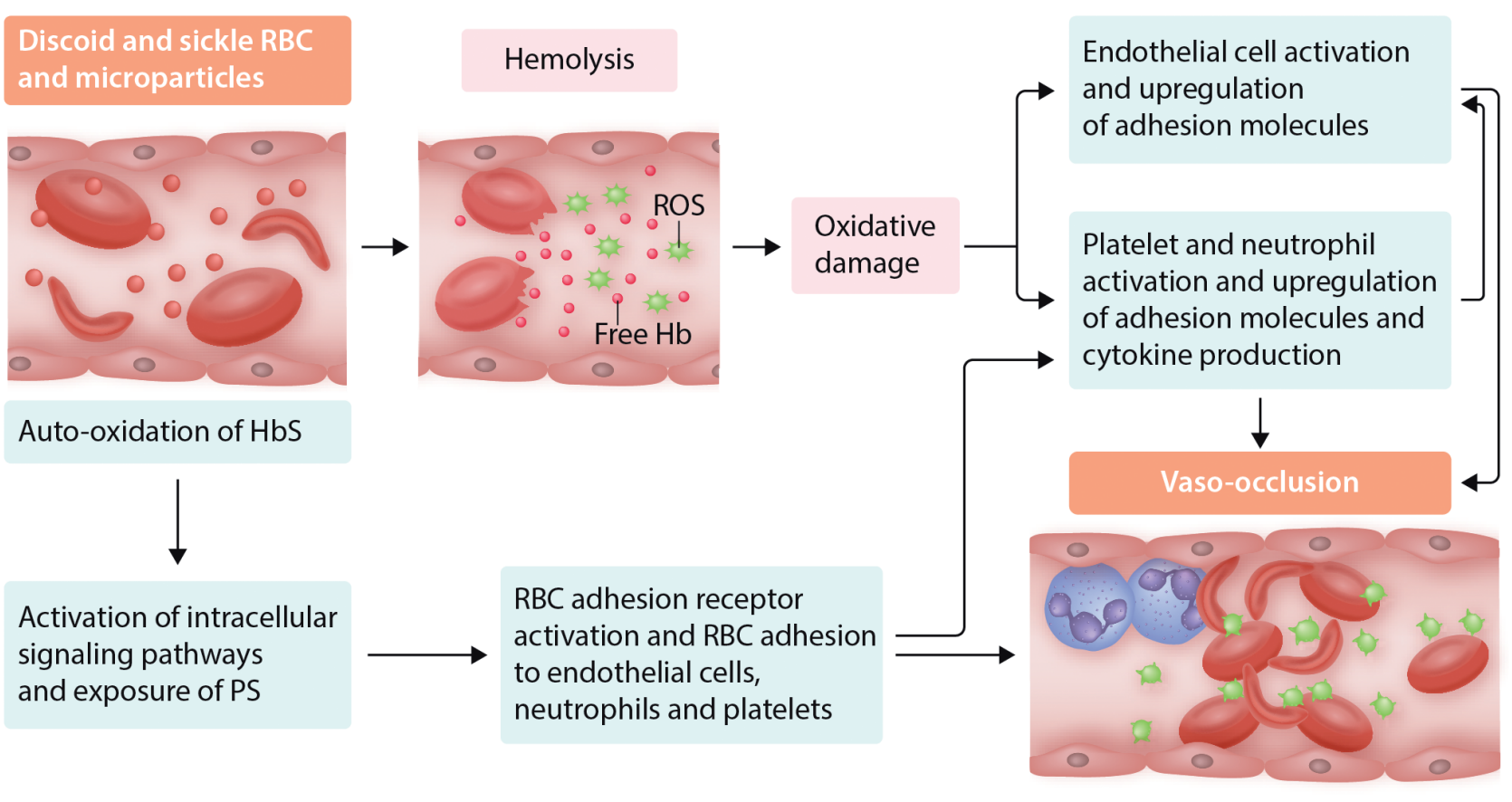

Figure 1. Pathophysiology of vaso-occlusion in sickle cell disease. Erythrocytes (RBC) containing predominantly hemoglobin (Hb) $\mathrm{S}$ (or HbS with another hemoglobin variant that participates in hemoglobin polymerization) circulate as biconcave discs as well as deformed "sickled" RBC; in addition, there is an abnormally increased number of RBC-derived microparticles in the circulation. Oxidative damage occurs both within the sickle RBC, due to HbS denaturation, instability and auto-oxidation, as well as in tissues in contact with the circulation, due to release of pro-oxidant substances (free $\mathrm{Hb}$ and reactive oxygen species [ROS]) by sickle RBC undergoing cell lysis (hemolysis). In the RBC, auto-oxidation of HbS promotes oxidation of $\beta$ Cys ${ }^{93}$, $\mathrm{Hb}$ dimerization and hemichrome formation. Sickle RBC also have retained mitochrondria, which can contribute oxidants that lead to membrane damage, and a diminished complement of anti-oxidant compounds, such as glutathione. Oxidative membrane changes and effect of oxidants on signaling pathways lead to both protein and lipid changes, including exposure of phosphatidylserine (PS) and upregulation of adhesion receptor activity, among other effects. In turn, hemolysis (with release of both Hb and ROS) leads to activation of endothelial cells as well as of cellular blood elements (leukocytes and platelets). In the post-capillary circulation, both sickle RBC and neutrophils adhere to endothelial cells as well as to each other. Platelets also participate in heterocellular aggregate formation. These interactions lead to vaso-occlusion in post-capillary venules. Obstruction of blood flow then results in further HbS deoxygenation,

can most avidly scavenge the $\bullet \mathrm{OCH}_{3}$ radical, in both aqueous and lipidic media, and also has weaker but physiologically relevant ability to trap $\bullet \mathrm{OOH}$ and $\bullet \mathrm{OOCH}_{3}$ radicals. ${ }^{14}$ Pyridoxamine also inhibits AGE formation due to its ability to bind to important enzymes responsible for oxidative reactions in the advanced stages of the protein glycation pathway.

Li et al. first determined whether sickle mice who receive pyridoxamine demonstrate clinically relevant beneficial effects. They showed that pyridoxamine reduced neutrophil recruitment to the cremaster venular wall of SCD mice after they were exposed to either hypoxia/reoxygenation or tumor necrosis factor- $\alpha$ (TNF$\alpha)$. Such treatment also improved survival of challenged mice. However, these effects did not appear to be via reduction in AGE, as pyridoxamine reduced endothelial cell and blood cell (neutrophil, platelet) activation states or adhesiveness without affecting the plasma levels of AGE or nitric oxide (NO). Moreover, pyridoxamine appeared equally effective in the presence or absence of hydroxyurea. In vitro studies also confirmed that pyridoxamine appears to have quite selective effects on neutrophils and platelets. It reduced neutrophil degranulation and decreased the surface amount of $\alpha \mathrm{M} \beta 2$ integrin, a receptor required for the interaction of neutrophils with endothelial cells and platelets, while it did not affect neutrophil L-selectin shedding and ROS production.
Furthermore, treatment of SCD mice or mouse platelets in vitro with pyridoxamine inhibited platelet aggregation and ATP secretion after exposure to thrombin or a collagen-related peptide (CRP, a glycoprotein VI-specific agonist) without affecting P-selectin. In vitro experiments with human neutrophils also showed that pyridoxamine reduced ROS generation but did not affect the surface amount of $\alpha \mathrm{M} \beta 2$ and L-selectin on neutrophils following agonist stimulation.

\section{What more needs to be done?}

Based on their results, Li et al. suggest that pyridoxamine should be investigated in human SCD to prevent both vaso-occlusion and possibly hemolysis-related organ damage. Although pyridoxamine did not seem to have its effect via reduction of AGE in sickle mice, its protective effect against oxidative challenges may be relevant. In diabetic rats, treatment with pyridoxamine produced a decline in oxidative stress parameters and ROS production. Given that oxidative damage is believed to be critical to the organ damage seen in both diabetes and SCD, further exploration of pyridoxamine in SCD is reasonable, despite the disappointing results in human diabetic patients despite promising data in animal models of diabetes. The article by $\mathrm{Li}$ et al. also offers interesting insights into where pyridoxamine may affect the many pathways leading to vaso-occlusion. It is disappointing, 
however, that they did not report effects of pyridoxamine on the root cause of vaso-occlusion, i.e., the sickle red blood cells (RBC). Especially at this time, when we know clearly that sickle RBC are deficient in anti-oxidant capacity ${ }_{15}^{15}$ and that oxidative pathways in RBC promote vasoocclusion, ${ }^{13}$ understanding the effects of anti-oxidant therapies on sickle RBC biology, the root cause of SCD, should be prioritized.

\section{References}

1. Telen MJ, Malik P, Vercellotti GM. Therapeutic strategies for sickle cell disease: towards a multi-agent approach. Nat Rev Drug Discov. 2019;18(2):139-158.

2. Li J, Jeong SY, Xiong B, et al. Repurposing pyridoxamine for therapeutic intervention of intravascular cell-cell interactions in mouse models of sickle cell disease. Haematologica. 2020;105(10):2407-2419.

3. Chen JL, Francis J. Pyridoxamine, advanced glycation inhibition, and diabetic nephropathy. J Am Soc Nephrol. 2012;23(1):6-8.

4. Lewis EJ, Greene T, Spitalewiz S, et al. Pyridorin in type 2 diabetic nephropathy. J Am Soc Nephrol. 2012;23(1):131-136.

5. Raval AD, Thakker D, Rangoonwala AN, Gor D, Walia R. Vitamin B and its derivatives for diabetic kidney disease. Cochrane Database Syst Rev. 2015;1:CD009403.
6. Nur E, Brandjes DP, Schnog JJ, et al. Plasma levels of advanced glycation end products are associated with haemolysis-related organ complications in sickle cell patients. Br J Haematol. 2010;151(1):62-69.

7. Niihara Y, Smith WR, Stark CW. A phase 3 trial of L-glutamine in sickle cell disease. N Engl J Med. 2018;379(19):1880.

8. Vichinsky E, Hoppe CC, Ataga KI, et al. A phase 3 randomized trial of voxelotor in sickle cell disease. N Engl J Med. 2019;381(6):509-519

9. Ataga KI, Kutlar A, Kanter J, et al. Crizanlizumab for the prevention of pain crises in sickle cell disease. N Engl J Med. 2017;376(5):429-439.

10. Belcher JD, Chen C, Nguyen J, et al. Control of oxidative stress and inflammation in sickle cell disease with the Nrf2 activator dimethyl fumarate. Antioxid Redox Signal. 2017;26(14):748-762.

11. Morris CR, Suh JH, Hagar W, et al. Erythrocyte glutamine depletion, altered redox environment, and pulmonary hypertension in sickle cell disease. Blood. 2008;111(1):402-410.

12. Nur E, Biemond BJ, Otten HM, Brandjes DP, Schnog JJ. Oxidative stress in sickle cell disease; pathophysiology and potential implications for disease management. Am J Hematol. 2011;86(6):484-489.

13. Thamilarasan M, Estupinan R, Batinic-Haberle I, Zennadi R. Mn porphyrins as a novel treatment targeting sickle cell NOXs to reverse and prevent acute vaso-occlusion in vivo. Blood Adv. 2020;4(11): 23722386

14. Ramis R, Ortega-Castro J, Caballero C, et al. How does pyridoxamine inhibit the formation of advanced glycation end products? The role of its primary antioxidant activity. Antioxidants (Basel). 2019;8(9):344.

15. Sangokoya C, Telen MJ, Chi JT. microRNA miR-144 modulates oxidative stress tolerance and associates with anemia severity in sickle cell disease. Blood. 2010;116(20):4338-4348.

\title{
ARQ531: the therapy that targets multiple pathways in acute myeloid leukemia
}

\author{
Charlotte Hellmich, ${ }^{1,2}$ Kristian Bowles ${ }^{1,2}$ and Stuart Rushworth ${ }^{1}$
}

${ }^{1}$ Norwich Medical School, University of East Anglia, Norwich Research Park, Norwich and 'Department of Haematology, Norfolk and Norwich University Hospitals NHS Trust, Norwich, UK

E-mail: STUART RUSHWORTH - s.rushworth@uea.ac.uk

doi:10.3324/haematol.2020.257022

S o far this century we have witnessed the introduction of a number of targeted therapies, developed through rational drug design, which have changed cancer treatment and resulted in improved outcomes for many patients, including those with a spectrum of chronic lymphoid and myeloid malignancies. ${ }^{1,2}$ However, despite improved understanding of the biology of acute myeloid leukemia (AML), similar scale benefits by targeting kinases and other intracellular and surface proteins have yet to be realized, and the prognosis for patients with AML remains poor. Moreover, cytotoxic drugs and therapies developed in the last century currently remain the backbone of AML treatment, and as AML primarily affects the elderly, many of whom are therefore frail with multiple co-morbidities, the clinical application of such curative therapies is somewhat limited. ${ }^{3}$ Furthermore, even in those fit enough for intensive chemotherapy, both relapse and treatment resistance are common, due to the aggressive nature of the disease. The search therefore continues for biology-driven targeted treatments for patients with AML which can be delivered to all, and at the same time increase remission rates, reduce relapses and prevent treatment resistance. The expectation is that these therapies will come from advances in the understanding of the biology of AML.
Tyrosine kinases are known to play a role in the tumorigenesis of both solid tumors and hematological malignancies and they therefore present a potential treatment target. ${ }^{4}$ In particular, Bruton tyrosine kinase (BTK) inhibitors have been shown to be effective in the treatment of a number of hematologic malignancies including chronic lymphocytic leukemia and lymphomas. ${ }^{5-7}$ BTK is a non-receptor tyrosine kinase with an important role in both normal and malignant hematopoiesis. ${ }^{8}$ Its expression and phosphorylation are high in AML and BTK inhibition with ibrutinib has been shown to have an anti-leukemic effect.' Moreover, many other tyrosine kinases have been shown to be activated in AML and hematologic malignancies including SYK, FLT3, MAPK, PI3K and AKT..$^{10,11}$ This knowledge supports further exploration of tyrosine kinase inibitors in the treatment of AML.

In this issue of Haematologica, Soncini et al. explore the potential role of ARO531, a reversible small molecule inhibitor of BTK and a number of other kinases, in the management of $\mathrm{AML}^{12}$ (Figure 1). BTK was shown to be constitutively active in a range of different subtypes of AML, suggesting that targeting it could have a broad clinical application in all patients with AML. ARQ531 was observed to have dose-dependent anti-leukemic activity by inducing apoptosis in both AML cell lines and primary AML cells. 